\title{
Thromboprophylaxis for Deep Vein Thrombosis and Pulmonary Embolism after Total Joint Arthroplasty in a Low Incidence Population
}

Kang-Il Kim, $\mathrm{MD}^{1}$, Dong-Geun Kang, $\mathrm{MD}^{1}$, Sumit S. Khurana, $\mathrm{MD}^{1}$, Sang-Hak Lee, MD ${ }^{1}$, Young-Joo Cho, $\mathrm{MD}^{1}$, and Dae-Kyung Bae, $\mathrm{MD}^{2}$

${ }^{1}$ Department of Orthopaedic Surgery, Center for Joint Diseases and Rheumatism, Kyung Hee University Hospital at Gangdong, Seoul; ${ }^{2}$ Department of Orthopaedic Surgery, Kyung Hee University School of Medicine, Seoul, Korea

Postoperative venous thromboembolism is one of the most serious complications following total joint arthroplasty. Pharmacological and mechanical prophylaxis methods are used to reduce the risk of postoperative symptomatic deep vein thrombosis and pulmonary embolism. Use of pharmacological prophylaxis requires a fine balance between the efficacy of the drug in preventing deep vein thrombosis and the adverse effects associated with the use of these drugs. In regions with a low prevalence of deep vein thrombosis such as Korea, there might be a question whether the benefits of using pharmacological prophylaxis outweigh the risks involved. The current article reviews the need for thromboprophylaxis, guidelines, problems with the guidelines, pharmacological prophylaxis use, and the current scenario of deep vein thrombosis, and discusses whether the use of pharmacological prophylaxis should be mandatory in low incidence populations.

Keywords: Total joint arthroplasty, Deep vein thrombosis, Pulmonary embolism, Thromboprophylaxis

\section{Introduction}

Postoperative venous thromboembolism (VTE) is one of the most serious complications following total joint arthroplasty (TJA $)^{1)}$. It is the duty of the operating surgeon to minimize the risk of occurrence of this complication and its associated morbidity and mortality. Numerous guidelines and recommendations suggest the use of various methods of thromboprophylaxis ${ }^{1-3)}$. Pharmacological and mechanical prophylaxis methods are used, either in isolation or in

Received January 16, 2013; Accepted April 10, 2013

Correspondence to: Kang-Il Kim, MD

Department of Orthopaedic Surgery, Center for Joint Diseases and Rheumatism, Kyung Hee University Hospital at Gangdong, 892

Dongnam-ro, Gangdong-gu, Seoul 134-727, Korea

Tel: +82-2-440-6151, Fax: +82-2-440-6296

E-mail: drkim@khu.ac.kr

This is an Open Access article distributed under the terms of the Creative Commons Attribution Non-Commercial License (http://creativecommons.org/licenses/by-nc/3.0/) which permits unrestricted non-commercial use, distribution, and reproduction in any medium, provided the original work is properly cited. combination, to reduce the risk of postoperative $\mathrm{VTE}^{1-3)}$. The use of pharmacological prophylaxis requires a fine balance between the efficacy of the drug in preventing deep-vein thrombosis (DVT) and the adverse effects associated with the use of these drugs $^{4)}$. In regions with a low prevalence of DVT such as Korea, do benefits of using pharmacological prophylaxis outweigh the risks involved?

In the current article, we review the need for thromboprophylaxis, the guidelines, problems with the guidelines and pharmacological prophylaxis, the current scenario of DVT and discuss whether the use of pharmacological prophylaxis should be mandatory in low incidence populations.

\section{Why Do We Need Thromboprophylaxis?}

Western literature reported $42 \%-57 \%$ incidence of total DVT following total hip arthroplasty (THA) and an incidence of $41 \%-85 \%$ following total knee arthroplasty (TKA) ${ }^{1}$. The corresponding pulmonary embolism (PE) rates are $0.9 \%-28 \%$ following THA and $1.5 \%-10 \%$ following TKA. Hence patients undergoing TJA are considered as a high risk 
group. Unfortunately, there is no way to identify which patient will develop postoperative VTE. Also fatal PE may be totally asymptomatic and may present as sudden death. Therefore, thromboprophylaxis use has been recommended for all patients undergoing TJA in Western population ${ }^{1-3)}$. Pharmacological thromboprophylaxis, in various randomized controlled trials, has shown to reduce the incidence of DVT-both distal and proximal $^{5-7)}$. Considering DVT as a surrogate marker for PE, thromboprophylaxis is presumed to decrease the incidence of fatal PE and sudden death correspondingly ${ }^{8}$. Timely prevention of DVT also reduces the cost associated with treating DVT and reduces morbidity associated with post-thrombotic syndrome and recurrent thrombosis.

However, variation exists in the rate of DVT occurrence with high incidence reported from the Western countries to low incidence reported in East Asia ${ }^{99}$. An estimated incidence across various countries in East Asia is shown in Table $1^{10-21)}$. Asian patients have traditionally been considered to have a low prevalence of DVT and $\mathrm{PE}^{9)}$. Moreover, a recent meta-analysis has reported that the incidence of VTE after knee arthropalsty without chemoprophylaxis was still low in Asians and there was no trend in changes of the incidence over time ${ }^{22}$. It has been understood that there is an association with the low prevalence of obesity, low frequency of hyperlipidemia, and the paucity of some genetic factors in this population ${ }^{18,23,24)}$. Activated protein $\mathrm{C}$ resistance, known as factor $\mathrm{V}$ Leiden, which is found in
$30 \%-50 \%$ patients with VTE in the Western population is rarely seen in Asians. The prothrombin promoter G20210A mutation, which is responsible for increased prothrombin levels and hence an increased risk of clotting, is again rarely seen in the Asian population. A low antithrombin III level increases the chances of clotting but most Asians have been found to have normal antithrombin III levels ${ }^{18,23,24)}$. Kim and $\mathrm{Kim}^{23)}$ suggested that the absence of thrombophilic polymorphisms with low clinical prothrombotic risk factors make occurrence of PE following THA very rare in the Asian population. Similar low rates are seen in Asians living in the West ${ }^{25}$. Difference in the dietary patterns (vegetarian diet) also has been suggested as one of the causes of the low incidence of $\mathrm{DVT}^{26}$.

Most Western countries have well established guidelines for thromboprohylaxis use after TJA such as the American College of Chest Physicians (ACCP) guidelines ${ }^{1)}$, American Academy of Orthopaedic Surgeons (AAOS) guidelines ${ }^{2}$, and National Institute for Health and Clinical Excellence (NICE) guidelines ${ }^{3)}$. No such guidelines exist in Asian countries and where they do exist they are not strictly adhered to ${ }^{27}$. Coupled with a low incidence are the risks involved with using pharmacological thromboprohylaxis ${ }^{28}$. Should it then be mandatory to prescribe routine thromboprophylaxis use following TJA in this part of the world?

Table 1. Prevalence of DVT without Thromboprophylaxis in East Asia (Since 2000)

\begin{tabular}{|c|c|c|c|c|c|c|c|c|}
\hline \multirow{2}{*}{ Author } & \multirow{2}{*}{ Year } & \multirow{2}{*}{ Country } & \multicolumn{2}{|r|}{ DVT screening } & \multirow{2}{*}{$\begin{array}{c}\text { No. of } \\
\text { patients }\end{array}$} & \multirow{2}{*}{$\begin{array}{c}\text { Total } \\
\text { DVT (\%) }\end{array}$} & \multirow{2}{*}{$\begin{array}{l}\text { Proximal } \\
\text { DVT (\%) }\end{array}$} & \multirow{2}{*}{$\mathrm{PE}(\%)$} \\
\hline & & & Day & Method & & & & \\
\hline \multicolumn{9}{|c|}{ Total hip arthroplasty } \\
\hline Kim et al. ${ }^{11)}$ & 2003 & Korea & $6-7$ & Venography & 200 & 24.0 & 14.0 & 0.0 \\
\hline Ko et al. ${ }^{12)}$ & 2003 & China & $6-8$ & USG doppler & 22 & 18.1 & 13.6 & 4.5 \\
\hline Yoo et al. ${ }^{14)}$ & 2009 & Korea & 7 & Venography/USG doppler & 221 & 10.4 & 1.8 & 0.5 \\
\hline Wong et al. ${ }^{15)}$ & 2011 & Singapore & 5 & USG doppler & 197 & 8.0 & 3.0 & 0.5 \\
\hline \multicolumn{9}{|c|}{ Total knee arthroplasty } \\
\hline Fong et al. ${ }^{16)}$ & 2000 & Singapore & 7 & USG doppler & 100 & 14.0 & 5.0 & 1.0 \\
\hline Chin et al. ${ }^{20)}$ & 2009 & Singapore & $5-7$ & USG doppler & 110 & 22.0 & 3.0 & 1.0 \\
\hline Kim et al. ${ }^{21)}$ & 2011 & Korea & 6 & USG doppler & 227 & 26.6 & 3.0 & 0.0 \\
\hline
\end{tabular}

DVT: deep vein thrombosis, PE: pulmonary embolism, USG: ultrasonography, NA: not available. 


\section{What Do the Guidelines Say?}

The new ACCP guidelines released in 2012 are based mostly on the results of fatal and symptomatic $\mathrm{PE}$ and deep vein thrombosis rather than overall VTE diagnosed by venographic screening tests $^{29)}$. The guideline recommends thromboprophylaxis use for patients undergoing TJA with low molecular weight heparin (LMWH), fondaparinux, apixaban, dabigatran, rivaroxaban, vitamin $\mathrm{K}$ antagonist, aspirin for a minimum of 10 to 14 days (grade 1B) rather than no prophylaxis ${ }^{1}$. Moreover, for asymptomatic patients following major orthopaedic surgery, it does not recommend duplex ultrasound screening before hospital discharge (grade 1B). The new AAOS guidelines "Preventing venous thromboembolic diseases in patients undergoing elective hip and knee arthroplasty” released in 2011 replaced the previous guideline, "Prevention of symptomatic $\mathrm{PE}$ in patients undergoing total hip and knee arthroplasty". It is based on a systematic review of related published articles. New guidelines contain 10 recommendations and 1 of them is graded as strong, 3 as moderate, 1 as weak, and 1 as inconclusive. Four recommendations are based on consensus ${ }^{30}$. The only strong recommendation is against routine use of duplex ultrasonography screening. The new guidelines suggest the use of pharmacologic agents and/or mechanical compressive device for prevention of VTE following elective TJA ${ }^{31}$. However, the guideline does not recommend any specific prophylactic agent or the duration of prophylaxis since it was not possible to determine differences in efficacy among these prophylactic agents with respect to symptomatic events. Meanwhile, both guidelines support combining a chemoprophylaxis with an intermittent pneumatic compression device for prevention of VTE in most patients. Therefore, combination of aspirin and a mechanical device is acceptable for patients undergoing knee arthroplasty ${ }^{32}$.

\section{Problems with Guidelines and Pharmacological Thromboprophylaxis Use}

\section{Complications of Anticoagulant Drugs}

Any pharmacological thromboprophylaxis use places the patient at risk of bleeding and its sequelae ${ }^{28)}$. The risks associated with thromboprophylaxis are varied and include hemorrhage, wound hematoma, persisting wound drainage, failure of wound healing, risk of infection and blood loss requiring transfusion ${ }^{33,34)}$. Bleeding is categorized as major if it is clinically overt or if it is retroperitoneal, intracranial, or intraocular in location, results in death or a serious or life-threatening clinical event or one requiring surgical intervention ${ }^{35)}$. Additionally a decrease in the hemoglobin level of $2.0 \mathrm{~g} / \mathrm{dL}(20 \mathrm{~g} / \mathrm{L})$ compared with the most recent preceding postoperative value, or transfusion of two or more units of blood products also constitutes as major bleeding. The bleeding is categorized as minor, if is overt, does not meet the criteria for major bleeding, and is associated with at least one of the following features: epistaxis lasting more than five minutes or requiring intervention, ecchymosis or hematoma larger than $5 \mathrm{~cm}$ at its greatest dimension, hematuria not associated with urinary catheter related trauma, gastrointestinal hemorrhage not related to intubation or placement of a nasogastric tube, wound hematoma or complications, or subconjunctival hemorrhage necessitating cessation of medication. The incidence of major bleeding associated with the use of chemoprophylaxis is reported as high as $4 \%-7.9 \%{ }^{36)}$ in the literature. There exists a direct correlation between the efficacy of the antithrombotic agents and their associated haemorrhagic complication rate. Injectable agents have higher bleeding rates as compared to the oral anticoagulants $^{37)}$. Burnett et al. ${ }^{33)}$ using the ACCP protocol of 10day course of enoxaparin $30 \mathrm{mg}$ twice daily in 290 patients undergoing TKA and THA showed an increased incidence of complications with $4.7 \%$ requiring readmission to hospital, $3.4 \%$ requiring reoperation and $5.1 \%$ requiring prolonged hospitalization for wound drainage and bleeding. As compared to warfarin, return to the operating room for wound complications occurred 3 times more frequently while symptomatic DVT still occurred in $3.8 \%$ patients and nonfatal PE in $1.3 \%$ patients. Wound drainage beyond 7 days was predictive of readmission and wound reoperation. Burnett et al. ${ }^{33)}$ suggested that surgical site complications requiring readmission and reoperation should be considered major complications. Patel et al. ${ }^{38)}$ evaluated 2,437 TJAs (both primary THAs and TKAs) and identified the use of LMWH as a risk factor for increased drain output and increased wound drainage after surgery. Also, each day of prolonged wound drainage increased the risk of infection by $42 \%$. Similar reports have been previously reported with the use of warfarin and unfractionated heparin ${ }^{36}$. Parvizi et al. ${ }^{39)}$ reviewed 78 cases that underwent revision for septic failure and demonstrated a direct correlation between administration of excessive anticoagulation and development of periprosthetic infection. A mean international normalized ratio (INR) of greater than 1.5 was found to be more prevalent in patients who developed postoperative wound complications and subsequent periprosthetic infection.

The adverse local effects of prophylactic agents are grouped together as minor bleeding events. Bleeding, blistering and ecchymosis till recently were not reported very often in the 
literature. Sachs et al. ${ }^{36)}$ reported increased local swelling and twice the infection rate in patients receiving 6 weeks of warfarin after TKA as compared to a cohort group receiving no prophylaxis with the same thromboembolic outcome in both groups. Keays et al. ${ }^{40)}$ reported that patients receiving enoxaparin following TKA had stiffness and delayed return of range of motion as compared to those receiving aspirin. Another side effect is increased incidence of transfusion secondary to prophylaxis use. Walsh et al. ${ }^{34)}$ evaluated the predictors of transfusion following THA and demonstrated that LMWH increased the need for transfusion 2.76 times. The occurrence of such complications after elective TJA due to the use of prophylactic agents is unacceptable to most surgeons.

\section{Timing of the Initiation of Prophylaxis}

There exists a controversy on the timing of initiation of the prophylaxis ${ }^{41}$. In Europe, LMWH prophylaxis is started 12 hours before surgery, while in North America, LMWH prophylaxis is initiated 12 hours after surgery to minimize the risk of intra and postoperative bleeding. Strebel et al. ${ }^{42)}$ showed comparable efficacy of both regimens of LMWH in a systematic review. Earlier initiation of prophylaxis has shown greater efficacy in preventing DVT but also causes a higher incidence of bleeding. For fondaparinux, it is recommended to start at either 6-8 hours after surgery or the next day since earlier dosing has shown to have greater bleeding ${ }^{43}$. The decision about when to initiate chemoprophylaxis should be based on the balanced efficacybleeding ratio of the prophylactic agents ${ }^{43)}$.

\section{Duration of Prophylaxis Use}

There is still no consensus on the duration of the use of prophylaxis. The previous version of ACCP guidelines recommended prophylaxis for a minimum duration of 10 days, which was extendable up to 35 days $^{1)}$, and previously the AAOS recommended LMWH/fondaparinux use for 7-12 days, whereas aspirin/warfarin use for 6 weeks ${ }^{2}$. Extended duration of prophylaxis using enoxaparin was shown effective following THA, but not following TKA ${ }^{44)}$. Only low molecular weight heparin has the evidence that longer term (28-35 days) administration shows more effectiveness than short term (7-10 days) use in prevention of symptomatic PE and DVT. However, the recently released new AAOS guidelines do not provide a specific duration for prophylaxis ${ }^{30}$. The work group rather recommends patients and physicians discuss the appropriate duration of prophylaxis for each individual situation ${ }^{30)}$. Newer drugs still do not have sufficient literature to recommend longer duration of prophylaxis ${ }^{2}$.

\section{Early Discharge}

Most guidelines recommend prophylaxis use for a minimum duration of 10 days, which is extendable up to $28-35$ days $^{1-3)}$. Extended duration of prophylaxis has been shown to be more effective in THA, though similar efficacy has not been shown following TKA ${ }^{44)}$. However, current hospital stay after elective TJA has been reduced to 3-7 days ${ }^{45}$. This implies most patients require post discharge prophylaxis. The additional cost of the drug and the need for a nursing staff for administration of the drug need to be borne in mind.

\section{Same Recommendations for THA and TKA}

Most guidelines have similar recommendations for both THA and TKA patients with minor differences. White et al. ${ }^{46)}$ analyzed records of 19,586 primary THA and 24,059 primary TKA in which $95 \%$ cases were found to have received similar frequency, type and duration of thromboprophylaxis. However, there exists a difference in the temporal pattern of DVT. Among patients who received prophylaxis, the median time to occurrence of symptomatic DVT was 7 days after TKA and 17 days after $\mathrm{THA}^{46}$. . Douketis et al. ${ }^{47)}$ showed that the prevalence of asymptomatic DVT was higher after TKA than THA (total DVT $38.8 \%$ vs.16.4\% and proximal DVT 7.6\% vs. 3.8\%). Post prophylaxis frequency of symptomatic VTE is higher after THA than TKA (2.5\% vs. $1.4 \%)$. The risk of VTE drops rapidly after TKA in contrast to THA where it remains elevated for a longer time ${ }^{47)}$. Hence similar prophylaxis regimens cannot be used for both these groups of patients.

\section{Can Asymptomatic DVT Be Taken as Marker for True DVT Prevalence?}

All prophylaxis use is based on the presumption that DVT and $\mathrm{PE}$ have the same pathophysiology and hence reduction in asymptomatic DVT would lead to reduction in the overall PE rates and sudden death rates ${ }^{8)}$. Can asymptomatic DVT be accepted as an appropriate surrogate marker for PE occurrence? While there is enough literature to support use of chemoprophylaxis for reducing asymptomatic DVT, there is no level one or level two evidence demonstrating a reduction of sudden death or PE rates $^{5-7}$. To assess a drug capable of reducing the risk of PE by $50 \%$ in hip arthroplasty patients, a minimum of 30,000-90,000 patients would be required ${ }^{48)}$. Currently, no such study exists for knee or hip arthroplasty patients and nor would be feasible. 


\section{Does Calf DVT Warrant Treatment?}

Distal DVT detected on venography or ultrasonography following TJA is stable and usually does not progress to PE. Howe et al. ${ }^{49)}$ showed asymptomatic calf DVT usually resolves by itself. Solis et al. ${ }^{50}$ showed that propagation of asymptomatic calf vein thrombosis after arthroplasty was not influenced by anticoagulation. Hence is pharmacological thromboprophylaxis indicated for asymptomatic calf DVT?

\section{Guidelines Are Not Routinely Followed}

Though numerous guidelines exist, there is still lack of adherence ${ }^{27)}$. Even in the US and UK where DVT prevalence is high, guidelines are not fully implemented ${ }^{51,52)}$. Controversies exist regarding the risk/benefit, cost/benefit and practicality of these guidelines ${ }^{4}$. Even among guidelines, there is a lack of consensus ${ }^{53}$. The difference between the ACCP and AAOS guidelines for DVT prophylaxis in the same American population stands a classic example although both new guidelines are getting similar than their previous versions. Can recommendations by one subspecialty (physicians) be binding on the other subspecialty (orthopaedic surgeons) ${ }^{54)}$ ?

\section{What Is the Current Scenario?}

\section{Early Mobilization Reduces DVT Occurrence}

Early mobilization has shown to reduce the incidence of DVT in patients undergoing TJA ${ }^{55-57)}$. Pearse et al. ${ }^{55)}$ in patients undergoing TKA, demonstrated a 30 fold reduction in DVT incidence with the use of early mobilization defined as starting to walk within 24 hours after surgery. Chandrasekaran et al. ${ }^{56)}$ further demonstrated in TKA patients that the distance mobilized on the first postoperative day directly correlated to the DVT incidence. The odds of developing DVT reduced by a third in patients who mobilized greater than $1 \mathrm{~m}$ on the first postoperative day and there was no DVT occurrence in patients who mobilized greater than $5 \mathrm{~m}$ on the first day after surgery. Similar results were shown by Husted et al. ${ }^{57)}$ in THA and TKA patients following a fast track program of early mobilization, shorter hospitalization and short duration of DVT prophylaxis. The new AAOS guidelines support early mobilization with consensus recommendation since there is an absence of reliable data. But it mentioned that early mobilization is of low cost, minimal risk to the patients, and consistent with current practice ${ }^{30)}$.

\section{Mechanical Methods of Prophylaxis Are Equally Effective}

Mechanical methods of prophylaxis like intermittent pneumatic compression devices and venous foot pump have been shown to be effective in reducing DVT prevalence ${ }^{58,59)}$. These devices increase venous outflow and/or reduce stasis within the leg veins thereby reducing the risk of clotting and help reduce the limb swelling. The primary advantage of using a mechanical method of prophylaxis is the lack of a side effect of bleeding. In fact, some authors have demonstrated that mechanical prophylaxis is equally efficacious as pharmacological thromboprophylaxis and have questioned the need for routine pharmacological thromboprophylaxis ${ }^{60)}$. Pitto et al. ${ }^{61)}$ demonstrated in a randomized controlled trial of 216 patients undergoing THA, use of venous foot pumps was effective and safer as compared to use of $\mathrm{LMWH}$. Yokote et al. ${ }^{60)}$ compared use of enoxaparin, fondaparinux and a placebo in combination with mechanical prophylaxis in 255 Japanese patients undergoing THA and demonstrated equal efficacy with no side effects in the placebo group using only mechanical prophylaxis.

\section{Epidural Anesthesia and Better Pain Management}

Use of epidural anesthesia and better pain management techniques has shown to reduce DVT occurrence ${ }^{62-64)}$. Sharrock et al. ${ }^{62)}$ showed that the use of epidural anesthesia reduced the incidence of both proximal and total DVT after both unilateral and one-stage bilateral TKA in 705 cases (541 patients). Similar efficacy of epidural anesthesia has also been shown in patients undergoing THA by Modig et al. ${ }^{63,64)}$ with significant reduction in both DVT and PE rates. Possible explanations are an increased blood circulation in the lower extremities, less tendency for intravascular clotting, and more efficient fibrinolysis seen in association with continuous epidural anesthesia. Use of newer pain management techniques like intravenous patientcontrolled analgesia, femoral nerve block, and local periarticular injections provide better pain control and patient satisfaction, reduce hospital stay, and improve function while minimizing complications ${ }^{65-67)}$. The new AAOS guidelines suggest the use of neuraxial (intrathecal, epidural, and spinal) anesthesia in patients following TJA to help limit blood loss, although the evidence support the neuraxial anesthesia does not affect the occurrence of VTE in the literature review ${ }^{30)}$.

\section{Use of Multimodal Thromboprophylaxis Methods}

Using a combination of various methods gives similar efficacy in DVT reduction while reducing side effects of the individual methods ${ }^{58,68-70)}$. Dorr et al. ${ }^{68)}$ used multimodal thromboprophylaxis in patients undergoing THA and TKA based on the preoperative risk assessment. The low risk patients 
were managed with aspirin, dipyridamole, or clopidogrel bisulfate along with the use of an intermittent pneumatic calf compression device. High risk patients were managed with LMWH or warfarin and intermittent calf compression. All patients were mobilized from bed within twenty-four hours after surgery. They concluded use of multimodal prophylaxis with risk stratification was efficacious while limiting adverse clinical outcomes secondary to thromboembolic, vascular, and bleeding complications. Similarly Daniel et al. ${ }^{58)}$ in 463 consecutive patients who underwent primary THA showed a lower incidence of VTE by using combination of aspirin, hypotensive epidural anaesthesia, elastic compression stockings, early mobilization and bilateral intermittent pneumatic calf compression device. By avoiding usage of a formal anticoagulant drug, they could minimize the bleeding complications.

\section{No Reduction in Overall PE Incidence}

$\mathrm{PE}$ rates have remained unchanged over the last $10-15$ years despite use of various drugs and guidelines ${ }^{5,71,72)}$. A metanalysis by Murray et al. ${ }^{71)}$ on patients undergoing THA showed $0.1 \%-0.2 \%$ fatal $\mathrm{PE}$ rate and $0.3 \%-0.4 \%$ death rate irrespective of prophylaxis use. Freedman et al. ${ }^{5}$ showed no difference in fatal PE rates or all-cause mortality with use of various prophylaxis methods in patients undergoing elective THA. The Scottish Arthroplasty Project shows a VTE rate of $2.08 \%$ that has remained unchanged between 1992 and 2001 despite improved prophylaxis usage during this period ${ }^{72}$. Khatod et al. ${ }^{73)}$, in a retrospective analysis of 30,000 patients undergoing TKA, showed only coumadin fared better than mechanical prophylaxis, whereas all other forms of chemical prophylaxis revealed no significant differences in the $\mathrm{PE}$ rates. With no difference in PE rates, are we adding more risks by additionally using anticoagulant prophylaxis? Sharrock et al. ${ }^{74}$ showed mortality was double $(0.4 \%)$ in patients receiving potent anticoagulants (including LMWH, ximelagatran, fondaparinux, rivaroxaban, and warfarin) versus patents receiving multimodal prophylaxis (regional anaesthesia, aspirin and pneumatic compression; $0.2 \%$ ). The incidence of PE was also significantly higher in those treated with potent anticoagulant agents versus multimodal treatment by a factor of up to $75 \%(0.6 \%$ vs. $0.35 \%)$. The conclusion drawn by Sharrock et al. ${ }^{74)}$ was that PE still occurs despite use of potent anticoagulant agents. Are we then exposing patients to a higher mortality risk postsurgery by using these potent prophylaxis drugs?

\section{Legal Issues}

With increased patient awareness, there are legal implications associated with the development of any complications including $\mathrm{DVT}^{75}$. There is a risk of misinterpreting the thromboprophylaxis guidelines as the minimum standards of care. This compels surgeons to prescribe pharmacological thromboprophylaxis to avoid any potential litigation. Would a surgeon working in a low DVT prevalence area and not using anticoagulant prophylaxis be considered negligent if a patient with no risk factor for DVT goes on to develop a DVT or fatal PE? Alternatively use of anticoagulant prophylaxis leading to wound drainage and periprosthetic infection in a low DVT prevalence area could again make the surgeon liable for his decision.

\section{Where Do We Stand?}

Use of any pharmacological thromboprophylaxis carries with it the risk of bleeding, hematoma formation, persisting wound drainage, and infection ${ }^{28,33)}$. There exists a need to seek a fine balance between antithrombotic efficacy and safety while prescribing any drug ${ }^{4}$. This becomes all the more important in a low DVT prevalence population since the benefits derived from prescription of an anticoagulant drug are few. In an ideal situation, pharmacological thromboprophylaxis should effectively reduce distal, proximal DVT and PE rates while minimizing side effects. Such an ideal strategy does not exist. Uncertainty of end point among various guidelines makes it difficult to define efficacy ${ }^{44,76)}$. Under-reporting of side effects makes it difficult to set margins of safety. Interplay of various risk factors in each patient makes risk assessment and quantification difficult. Factors related to drug use, dosage and timing of administration, add further variables. Mechanical methods of prophylaxis are proving to be equally efficacious ${ }^{58,59)}$. Considering the already low proximal DVT and PE rate, it is still debatable to apply the Western protocol of pharmacological thromboprophylaxis to every patient undergoing TJA in low incidence populations such as Korea ${ }^{22)}$.

Though the general consensus is patients with proximal DVT should be treated with anticoagulants, Kim et al. ${ }^{11)}$ reported that all thrombi regardless of their site or size resolve completely and spontaneously without causing any PE. Hence they do not routinely use anticoagulants for prophylaxis in their patients undergoing TJA. However, considering a fatal PE although it has been reported extremely rare in Asia, such a policy of no use of anticoagulant prophylaxis could be dangerous ${ }^{77,78)}$. What is required is thus a judicious use of pharmacological thromboprophylaxis which does not compromise patient safety. 


\section{What Are the Possible Solutions?}

\section{Risk Stratification}

Because of the numerous variables involved, a single regimen or protocol cannot be effectively used for all patients. Using a group specific prophylaxis approach (i.e., for all patients undergoing TJA) is easier to follow ${ }^{1)}$. However this alone does not solve the problem as all patients undergoing TJA are classified as a high risk group. Hence a need to add a risk stratified approach. Each patient needs a preoperative risk assessment done based on the individual predisposing factors and the procedure being performed (THA/TKA). Such formal risk assessment models have been proposed but need to be modified based on the regional VTE prevalence and then subsequently validated before routine use.

\section{Modifications in Preoperative, Intraoperative, and Postoperative Care}

Preoperatively, all patients should be assessed for risk of DVT and risk of major bleeding as suggested by the AAOS guidelines ${ }^{2)}$. Regional anaesthesia should be preferably used unless contraindicated ${ }^{63,64)}$. Meticulous intraoperative haemostasis and 'water tight' closure of arthrotomy should be done to prevent wound drainage and haematoma formation ${ }^{4}$. Mechanical methods of prophylaxis should be used both intra- and postoperatively since they have few side effects ${ }^{58-60)}$. Effort should be made to mobilize patients postoperatively as soon as possible within constraints of patient safety and comfort ${ }^{55-57)}$. Better pain management and use of multimodal techniques should be advocated to reduce patient discomfort and facilitate early ambulation ${ }^{65-70,79)}$. Patients who have been immobilized or have restricted mobility due to medical or surgical reasons like chest infection, postoperative ileus or electrocardiogram, changes should be continued on prophylaxis $^{2)}$.

\section{Assessment of Regional DVT Prevalence}

Any guideline/protocol needs to be modified according to the regional DVT and PE prevalence. This would require an accurate assessment of the current DVT and PE rates in each population subgroup $^{21)}$. This would also help to minimize variations occurring due to the different postoperative practices being followed.

\section{Randomized Controlled Trials}

Further randomized controlled trials are needed to identify the risk factors predisposing to DVT and how these risk factors contribute to the pathophysiology of $\mathrm{DVT}^{22)}$. Newer drugs need to be compared for their efficacy and side effects with existing drugs and regimens being followed. Existing drugs need to be further studied in individual population groups to balance the efficacy-safety ratio and to assess the dose-duration relationship. Emphasis needs to be placed on accurate and detailed reporting of minor bleeding events rather than grouping all these events into one subgroup. Wound related complications should be considered major complications as they carry a high risk of infection.

\section{Auditing and Reporting of Data}

Local hospital committees should be created to deal with the problem of VTE, formation and implementation of protocols of thromboprophylaxis, and the auditing of results ${ }^{80,81)}$. Meticulous data recording needs to be emphasized. Collected data needs to be shared with other regions to help formulate national standards and guidelines for DVT prevention.

\section{Patient Education}

All patients should be educated about common symptoms of DVT and PE. The importance of continuous mobilization after discharge from hospital needs to be understood rather than enforced. Physical and occupational therapy teams should collaborate with the family of the patient so that the exercise program could be continued after discharge in the home environment. This would not only help in reducing DVT and PE incidence but also improve overall outcome of TJA.

Successful implementation of these strategies would help us to decide which patients require pharmacological thromboprophylaxis following TJA in a low DVT incidence population. Patients with no risk factors and those who can be mobilized early could be managed with the use of mechanical methods of prophylaxis alone. Patients with risk factors for DVT should have a risk assessment and based on that should be advised about multimodal thromboprophylaxis so that the side effects could be minimized. Formulating such a policy would not only reduce VTE prevalence but also improve the quality of patient care.

\section{Conflict of Interest}

No potential conflict of interest relevant to this article was reported. 


\section{References}

1. Geerts WH, Bergqvist D, Pineo GF, Heit JA, Samama CM, Lassen MR, Colwell CW; American College of Chest Physicians. Prevention of venous thromboembolism: American College of Chest Physicians Evidence-Based Clinical Practice Guidelines (8th Edition). Chest. 2008;133(6 Suppl):381S-453S.

2. Lachiewicz PF. Prevention of symptomatic pulmonary embolism in patients undergoing total hip and knee arthroplasty: clinical guideline of the American Academy of Orthopaedic Surgeons. Instr Course Lect. 2009;58:795-804.

3. Hill J, Treasure T. Reducing the risk of venous thromboembolism (deep vein thrombosis and pulmonary embolism) in inpatients having surgery: summary of NICE guidance. BMJ. 2007;334:1053-4.

4. Cushner FD, Nett MP. Unanswered questions, unmet needs in venous thromboprophylaxis. Orthopedics. 2009;32(12 Suppl):62-6.

5. Freedman KB, Brookenthal KR, Fitzgerald RH Jr, Williams S, Lonner JH. A meta-analysis of thromboembolic prophylaxis following elective total hip arthroplasty. J Bone Joint Surg Am. 2000;82:929-38.

6. Cohen AT, Bailey CS, Alikhan R, Cooper DJ. Extended thromboprophylaxis with low molecular weight heparin reduces symptomatic venous thromboembolism following lower limb arthroplasty: a meta-analysis. Thromb Haemost. 2001;85:940-1.

7. Huisman MV, Quinlan DJ, Dahl OE, Schulman S. Enoxaparin versus dabigatran or rivaroxaban for thromboprophylaxis after hip or knee arthroplasty: results of separate pooled analyses of phase III multicenter randomized trials. Circ Cardiovasc Qual Outcomes. 2010;3:652-60.

8. Warwick D. New concepts in orthopaedic thromboprophylaxis. J Bone Joint Surg Br. 2004;86:788-92.

9. Kanchanabat B, Stapanavatr W, Meknavin S, Soorapanth C, Sumanasrethakul C, Kanchanasuttirak P. Systematic review and meta-analysis on the rate of postoperative venous thromboembolism in orthopaedic surgery in Asian patients without thromboprophylaxis. Br J Surg. 2011;98:1356-64.

10. Fujita S, Hirota S, Oda T, Kato Y, Tsukamoto Y, Fuji T. Deep venous thrombosis after total hip or total knee arthroplasty in patients in Japan. Clin Orthop Relat Res. 2000;(375):16874.

11. Kim YH, Oh SH, Kim JS. Incidence and natural history of deep-vein thrombosis after total hip arthroplasty: a prospective and randomised clinical study. J Bone Joint Surg Br. 2003;85:661-5.

12. Ko PS, Chan WF, Siu TH, Khoo J, Wu WC, Lam JJ. Deep venous thrombosis after total hip or knee arthroplasty in a "low-risk" Chinese population. J Arthroplasty. 2003;18:1749.

13. Sudo A, Sano T, Horikawa K, Yamakawa T, Shi D, Uchida A. The incidence of deep vein thrombosis after hip and knee arthroplasties in Japanese patients: a prospective study. J Orthop Surg (Hong Kong). 2003;11:174-7.

14. Yoo MC, Cho YJ, Ghanem E, Ramteke A, Kim KI. Deep vein thrombosis after total hip arthroplasty in Korean patients and D-dimer as a screening tool. Arch Orthop Trauma Surg. 2009;129:887-94.

15. Wong KL, Daguman R, Lim K, Shen L, Lingaraj K. Incidence of deep vein thrombosis following total hip arthroplasty: a Doppler ultrasonographic study. J Orthop Surg (Hong Kong). 2011;19:50-3.

16. Fong YK, Ruban P, Yeo SJ, Lee BP, Lo NN, Seow KH, Ng SC. Use of low molecular weight heparin for prevention of deep vein thrombosis in total knee arthroplasty: a study of its efficacy in an Asian population. Ann Acad Med Singapore. 2000;29:439-41.

17. Wang CJ, Wang JW, Weng LH, Huang CC, Yu PC. Clinical significance of muscular deep-vein thrombosis after total knee arthroplasty. Chang Gung Med J. 2007;30:41-6.

18. Kim YH, Yoo JH, Kim JS. Factors leading to decreased rates of deep vein thrombosis and pulmonary embolism after total knee arthroplasty. J Arthroplasty. 2007;22:974-80.

19. Chen CJ, Wang CJ, Huang CC. The value of D-dimer in the detection of early deep-vein thrombosis after total knee arthroplasty in Asian patients: a cohort study. Thromb J. 2008;6:5.

20. Chin PL, Amin MS, Yang KY, Yeo SJ, Lo NN. Thromboembolic prophylaxis for total knee arthroplasty in Asian patients: a randomised controlled trial. J Orthop Surg (Hong Kong). 2009;17:1-5.

21. Kim KI, Cho KY, Jin W, Khurana SS, Bae DK. Recent Korean perspective of deep vein thrombosis after total knee arthroplasty. J Arthroplasty. 2011;26:1112-6.

22. Lee WS, Kim KI, Lee HJ, Kyung HS, Seo SS. The incidence of pulmonary embolism and deep vein thrombosis after knee arthroplasty in Asians remains low: a meta-analysis. Clin Orthop Relat Res. 2013;471:1523-32.

23. Kim YH, Kim JS. The 2007 John Charnley Award. Factors leading to low prevalence of DVT and pulmonary embolism 
after THA: analysis of genetic and prothrombotic factors. Clin Orthop Relat Res. 2007;465:33-9.

24. Kim YH, Kim VE. Factors leading to low incidence of deep vein thrombosis after cementless and cemented total knee arthroplasty. Clin Orthop Relat Res. 1991;(273):119-24.

25. Stein PD, Kayali F, Olson RE, Milford CE. Pulmonary thromboembolism in Asians/Pacific Islanders in the United States: analysis of data from the National Hospital Discharge Survey and the United States Bureau of the Census. Am J Med. 2004;116:435-42.

26. Leizorovicz A; SMART Venography Study Steering Committee. Epidemiology of post-operative venous thromboembolism in Asian patients: results of the SMART venography study. Haematologica. 2007;92:1194-200.

27. Warwick D, Dahl OE, Fisher WD; International Surgical Thrombosis Forum. Orthopaedic thromboprophylaxis: limitations of current guidelines. J Bone Joint Surg Br. 2008;90:127-32.

28. Schulman S, Beyth RJ, Kearon C, Levine MN; American College of Chest Physicians. Hemorrhagic complications of anticoagulant and thrombolytic treatment: American College of Chest Physicians Evidence-Based Clinical Practice Guidelines (8th Edition). Chest. 2008;133(6 Suppl):257S98S.

29. Falck-Ytter Y, Francis CW, Johanson NA, Curley C, Dahl OE, Schulman S, Ortel TL, Pauker SG, Colwell CW Jr; American College of Chest Physicians. Prevention of VTE in orthopedic surgery patients: Antithrombotic Therapy and Prevention of Thrombosis, 9th ed: American College of Chest Physicians Evidence-Based Clinical Practice Guidelines. Chest. 2012;141(2 Suppl):e278S-325S.

30. Mont MA, Jacobs JJ, Boggio LN, Bozic KJ, Della Valle CJ, Goodman SB, Lewis CG, Yates AJ Jr, Watters WC 3rd, Turkelson CM, Wies JL, Donnelly P, Patel N, Sluka P; AAOS. Preventing venous thromboembolic disease in patients undergoing elective hip and knee arthroplasty. J Am Acad Orthop Surg. 2011;19:768-76.

31. Lieberman JR. The new AAOS clinical practice guidelines on venous thromboembolic prophylaxis: how to adapt them to your practice. J Am Acad Orthop Surg. 2011;19:717-21.

32. Lieberman JR. American College of Chest Physicians evidence-based guidelines for venous thromboembolic prophylaxis: the guideline wars are over. J Am Acad Orthop Surg. 2012;20:333-5.

33. Burnett RS, Clohisy JC, Wright RW, McDonald DJ, Shively RA, Givens SA, Barrack RL. Failure of the American College of Chest Physicians-1A protocol for lovenox in clinical outcomes for thromboembolic prophylaxis. J Arthroplasty. 2007;22:317-24.

34. Walsh M, Preston C, Bong M, Patel V, Di Cesare PE. Relative risk factors for requirement of blood transfusion after total hip arthroplasty. J Arthroplasty. 2007;22:1162-7.

35. Schulman S, Angerås U, Bergqvist D, Eriksson B, Lassen MR, Fisher W; Subcommittee on Control of Anticoagulation of the Scientific and Standardization Committee of the International Society on Thrombosis and Haemostasis. Definition of major bleeding in clinical investigations of antihemostatic medicinal products in surgical patients. J Thromb Haemost. 2010;8:202-4.

36. Sachs RA, Smith JH, Kuney M, Paxton L. Does anticoagulation do more harm than good? A comparison of patients treated without prophylaxis and patients treated with lowdose warfarin after total knee arthroplasty. J Arthroplasty. 2003;18:389-95.

37. Fitzgerald RH Jr, Spiro TE, Trowbridge AA, Gardiner GA Jr, Whitsett TL, O'Connell MB, Ohar JA, Young TR; Enoxaparin Clinical Trial Group. Prevention of venous thromboembolic disease following primary total knee arthroplasty: a randomized, multicenter, open-label, parallelgroup comparison of enoxaparin and warfarin. J Bone Joint Surg Am. 2001;83:900-6.

38. Patel VP, Walsh M, Sehgal B, Preston C, DeWal H, Di Cesare PE. Factors associated with prolonged wound drainage after primary total hip and knee arthroplasty. J Bone Joint Surg Am. 2007;89:33-8.

39. Parvizi J, Ghanem E, Joshi A, Sharkey PF, Hozack WJ, Rothman RH. Does "excessive" anticoagulation predispose to periprosthetic infection? J Arthroplasty. 2007;22(6 Suppl 2):24-8.

40. Keays AC, Mason M, Keays SL, Newcombe PA. The effect of anticoagulation on the restoration of range of motion after total knee arthroplasty: enoxaparin versus aspirin. J Arthroplasty. 2003;18:180-5.

41. Raskob GE, Hirsh J. Controversies in timing of the first dose of anticoagulant prophylaxis against venous thromboembolism after major orthopedic surgery. Chest. 2003;124(6 Suppl):379S-85S.

42. Strebel N, Prins M, Agnelli G, Büller HR. Preoperative or postoperative start of prophylaxis for venous thromboembolism with low-molecular-weight heparin in elective hip surgery? Arch Intern Med. 2002;162:1451-6.

43. Kwong LM, Muntz JE. Thromboprophylaxis dosing: the 
relationship between timing of first administration, efficacy, and safety. Am J Orthop (Belle Mead NJ). 2002;31(11 Suppl):16-20.

44. Eikelboom JW, Quinlan DJ, Douketis JD. Extended-duration prophylaxis against venous thromboembolism after total hip or knee replacement: a meta-analysis of the randomised trials. Lancet. 2001;358:9-15.

45. Kearon C. Duration of venous thromboembolism prophylaxis after surgery. Chest. 2003;124(6 Suppl):386S-92S.

46. White RH, Romano PS, Zhou H, Rodrigo J, Bargar W. Incidence and time course of thromboembolic outcomes following total hip or knee arthroplasty. Arch Intern Med. 1998;158:1525-31.

47. Douketis JD, Eikelboom JW, Quinlan DJ, Willan AR, Crowther MA. Short-duration prophylaxis against venous thromboembolism after total hip or knee replacement: a meta-analysis of prospective studies investigating symptomatic outcomes. Arch Intern Med. 2002;162:146571.

48. Lie SA, Engesaeter LB, Havelin LI, Furnes O, Vollset SE. Early postoperative mortality after 67,548 total hip replacements: causes of death and thromboprophylaxis in 68 hospitals in Norway from 1987 to 1999. Acta Orthop Scand. 2002;73:392-9.

49. Howe CT, Kakkar VV, Flanc C, Clarke MB. The natural history of deep-vein thrombosis. Br J Surg. 1969;56:625.

50. Solis MM, Ranval TJ, Nix ML, Eidt JF, Nelson CL, Ferris EJ, Lavender RC, Barnes RW. Is anticoagulation indicated for asymptomatic postoperative calf vein thrombosis? J Vasc Surg. 1992;16:414-9.

51. Amin AN, Stemkowski S, Lin J, Yang G. Inpatient thromboprophylaxis use in U.S. hospitals: adherence to the seventh American College of Chest Physician's recommendations for at-risk medical and surgical patients. J Hosp Med. 2009;4:E15-21.

52. Sharif KM, Rehman SF, Nunn T, Bagga TK. Implementation of the NICE guidelines for venous thromboprophylaxis: a national survey of hip surgeons. Hip Int. 2009;19:58-63.

53. Struijk-Mulder MC, Ettema HB, Verheyen CC, Buller HR. Comparing consensus guidelines on thromboprophylaxis in orthopedic surgery. J Thromb Haemost. 2010;8:678-83.

54. Callaghan JJ, Dorr LD, Engh GA, Hanssen AD, Healy WL, Lachiewicz PF, Lonner JH, Lotke PA, Ranawat CS, Ritter MA, Salvati EA, Sculco TP, Thornhill TS; American Colleg of Chest Physicians. Prophylaxis for thromboembolic disease: recommendations from the American College of Chest Physicians: are they appropriate for orthopaedic surgery? J Arthroplasty. 2005;20:273-4.

55. Pearse EO, Caldwell BF, Lockwood RJ, Hollard J. Early mobilisation after conventional knee replacement may reduce the risk of postoperative venous thromboembolism. J Bone Joint Surg Br. 2007;89:316-22.

56. Chandrasekaran S, Ariaretnam SK, Tsung J, Dickison D. Early mobilization after total knee replacement reduces the incidence of deep venous thrombosis. ANZ J Surg. 2009;79:526-9.

57. Husted H, Otte KS, Kristensen BB, Orsnes T, Wong C, Kehlet H. Low risk of thromboembolic complications after fast-track hip and knee arthroplasty. Acta Orthop. 2010;81:599-605.

58. Daniel J, Pradhan A, Pradhan C, Ziaee H, Moss M, Freeman J, McMinn DJ. Multimodal thromboprophylaxis following primary hip arthroplasty: the role of adjuvant intermittent pneumatic calf compression. J Bone Joint Surg Br. 2008;90:562-9.

59. Pitto RP, Young S. Foot pumps without graduated compression stockings for prevention of deep-vein thrombosis in total joint replacement: efficacy, safety and patient compliance. A comparative, prospective clinical trial. Int Orthop. 2008;32:331-6.

60. Yokote R, Matsubara M, Hirasawa N, Hagio S, Ishii K, Takata C. Is routine chemical thromboprophylaxis after total hip replacement really necessary in a Japanese population? J Bone Joint Surg Br. 2011;93:251-6.

61. Pitto RP, Hamer H, Heiss-Dunlop W, Kuehle J. Mechanical prophylaxis of deep-vein thrombosis after total hip replacement a randomised clinical trial. J Bone Joint Surg Br. 2004;86:639-42.

62. Sharrock NE, Haas SB, Hargett MJ, Urquhart B, Insall JN, Scuderi G. Effects of epidural anesthesia on the incidence of deep-vein thrombosis after total knee arthroplasty. J Bone Joint Surg Am. 1991;73:502-6.

63. Modig J, Borg T, Karlstrom G, Maripuu E, Sahlstedt B. Thromboembolism after total hip replacement: role of epidural and general anesthesia. Anesth Analg. 1983;62:17480.

64. Modig J. The role of lumbar epidural anaesthesia as antithrombotic prophylaxis in total hip replacement. Acta Chir Scand. 1985;151:589-94.

65. Ranawat AS, Ranawat CS. Pain management and accelerated rehabilitation for total hip and total knee arthroplasty. J Arthroplasty. 2007;22(7 Suppl 3):12-5. 
66. Paul JE, Arya A, Hurlburt L, Cheng J, Thabane L, Tidy A, Murthy Y. Femoral nerve block improves analgesia outcomes after total knee arthroplasty: a meta-analysis of randomized controlled trials. Anesthesiology. 2010;113:1144-62.

67. Busch CA, Whitehouse MR, Shore BJ, MacDonald SJ, McCalden RW, Bourne RB. The efficacy of periarticular multimodal drug infiltration in total hip arthroplasty. Clin Orthop Relat Res. 2010;468:2152-9.

68. Dorr LD, Gendelman V, Maheshwari AV, Boutary M, Wan Z, Long WT. Multimodal thromboprophylaxis for total hip and knee arthroplasty based on risk assessment. J Bone Joint Surg Am. 2007;89:2648-57.

69. Maheshwari AV, Blum YC, Shekhar L, Ranawat AS, Ranawat CS. Multimodal pain management after total hip and knee arthroplasty at the Ranawat Orthopaedic Center. Clin Orthop Relat Res. 2009;467:1418-23.

70. Salvati EA. Multimodal prophylaxis of venous thrombosis. Am J Orthop (Belle Mead NJ). 2002;31(9 Suppl):4-11.

71. Murray DW, Britton AR, Bulstrode CJ. Thromboprophylaxis and death after total hip replacement. J Bone Joint Surg Br. 1996;78:863-70.

72. Howie C, Hughes H, Watts AC. Venous thromboembolism associated with hip and knee replacement over a ten-year period: a population-based study. J Bone Joint Surg Br. 2005;87:1675-80.

73. Khatod M, Inacio MC, Bini SA, Paxton EW. Pulmonary embolism prophylaxis in more than 30,000 total knee arthroplasty patients: is there a best choice? J Arthroplasty. 2012;27:167-72.

74. Sharrock NE, Gonzalez Della Valle A, Go G, Lyman S, Salvati EA. Potent anticoagulants are associated with a higher all-cause mortality rate after hip and knee arthroplasty. Clin
Orthop Relat Res. 2008;466:714-21.

75. Kessler DP, Summerton N, Graham JR. Effects of the medical liability system in Australia, the UK, and the USA. Lancet. 2006;368:240-6.

76. Lachiewicz PF. Comparison of ACCP and AAOS guidelines for VTE prophylaxis after total hip and total knee arthroplasty. Orthopedics. 2009;32(12 Suppl):74-8.

77. Piovella F, Wang CJ, Lu H, Lee K, Lee LH, Lee WC, Turpie AG, Gallus AS, Planès A, Passera R, Rouillon A; AIDA investigators. Deep-vein thrombosis rates after major orthopedic surgery in Asia: an epidemiological study based on postoperative screening with centrally adjudicated bilateral venography. J Thromb Haemost. 2005;3:2664-70.

78. Leizorovicz A, Turpie AG, Cohen AT, Wong L, Yoo MC, Dans A; SMART Study Group. Epidemiology of venous thromboembolism in Asian patients undergoing major orthopedic surgery without thromboprophylaxis: the SMART study. J Thromb Haemost. 2005;3:28-34.

79. Parvataneni HK, Shah VP, Howard H, Cole N, Ranawat AS, Ranawat CS. Controlling pain after total hip and knee arthroplasty using a multimodal protocol with local periarticular injections: a prospective randomized study. J Arthroplasty. 2007;22(6 Suppl 2):33-8.

80. Cohn SL. Prophylaxis of venous thromboembolism in the US: improving hospital performance. J Thromb Haemost. 2009;7:1437-45.

81. Grimshaw JM, Shirran L, Thomas R, Mowatt G, Fraser C, Bero L, Grilli R, Harvey E, Oxman A, O'Brien MA. Changing provider behavior: an overview of systematic reviews of interventions. Med Care. 2001;39(8 Suppl 2):II245. 